\title{
ARTICLE
}

\section{A novel biphenyl compound IMB-S7 ameliorates hepatic fibrosis in BDL rats by suppressing Sp1-mediated integrin $\alpha \mathrm{v}$ expression}

\author{
Na Zhang ${ }^{1}$, Shuang-shuang Zhao ${ }^{1,2,3}$, Yi-xuan Zhang ${ }^{1}$, Yu-cheng Wang ${ }^{1}$, Rong-guang Shao ${ }^{1}$, Ju-xian Wang ${ }^{1}$ and Hong-wei He ${ }^{1}$
}

\begin{abstract}
Chronic tissue injury with fibrosis results in the disruption of tissue architecture, organ dysfunction, and eventual organ failure. Therefore, the development of effective antifibrotic drugs is urgently required. IMB-S7 is novel biphenyl compound derived from bifendate (biphenyldicarboxylate) that is used for the treatment of chronic hepatitis in China. In the current study we investigated the potential of IMB-S7 as an antihepatic fibrosis agent. In bile duct ligation (BDL) rat model, oral administration of IMBS7 (400 mg $\mathrm{kg}^{-1} \cdot \mathrm{d}^{-1}$, for 14 days) significantly ameliorated BDL-induced liver necrosis, bile duct proliferation, and collagen accumulation. We then showed that IMB-S7 treatment markedly suppressed the TGF- $\beta / S$ mad pathway in human hepatic stellate cell line LX2 and mouse primary HSCs, as well as in liver samples of BDL rats, thus inhibiting the transcription of most fibrogenesisassociated genes, including TGF- $\beta 1, C O L 1 A 1$, and ACTA2. Furthermore, IMB-S7 treatment significantly suppressed the expression of integrin av at the mRNA and protein levels in TGF- $\beta$-treated LX2 cells and liver samples of BDL rats. Using integrin av overexpression and silencing, we demonstrated that integrin av activity correlated positively with the activation of TGF- $\beta / S m a d$ pathway. Based on dual luciferase assay and DNA affinity precipitation assay, we revealed that IMB-S7 inactivated integrin av through competitively inhibiting the binding of Sp1, a transcription factor, to the integrin av (ITGAV promoter (-173/-163 bp). These results suggest that IMB-S7 inhibits HSCs activation and liver fibrosis through Sp1-integrin av signaling, and IMB-S7 may be a promising candidate to combat hepatic fibrosis in the future.
\end{abstract}

Keywords: bifendate; IMB-S7; hepatic fibrosis; integrin av; Sp1; TGF- $\beta / S m a d$ pathway; bile duct ligation; LX2 cells

Acta Pharmacologica Sinica (2020) 41:661-669; https://doi.org/10.1038/s41401-019-0325-6

\section{INTRODUCTION}

Liver fibrosis, which is characterized by excessive extracellular matrix (ECM) deposition resulting from chronic liver injury of different etiologies, represents a major health problem worldwide. The development of fibrosis is the first step toward the progression to cirrhosis and its complications, including portal hypertension, organ failure, and hepatocellular carcinoma [1]. Nonalcoholic steatohepatitis, viral infection, and alcohol are the most common causes of hepatic fibrosis, and Asians have a high prevalence of this condition due to frequent infection of viral hepatitis B or C [2]. Hepatic stellate cells (HSCs) reside in the space of Disse in the liver and are the predominant fibrogenic cell type that produces ECM in response to various injuries. The inhibition of HSC activation is proposed as a therapeutic strategy for hepatic fibrosis treatment. The HSC activation process is induced by various stimulatory factors. Among them, TGF- $\beta 1$ plays a central role. In this regard, abolishing TGF- $\beta 1$ synthesis or TGF- $\beta 1$ mediated signaling pathways has been shown to decrease fibrosis that has been implicated in experimental models and patients $[3,4]$.
Integrins, a family of transmembrane cell adhesion molecules, are composed of noncovalently linked heterodimers of 18 asubunits and $8 \beta$-subunits that form at least 24 combinations in mammals [5]. The main roles of integrins are promoting the attachment of cells to ECM proteins and transporting signals from the ECM to the cells and vice versa [6]. Thus, integrins provide a major node of communication between the ECM, inflammatory cells, fibroblasts, and parenchymal cells. As such, integrins are intimately involved in the initiation, maintenance, and resolution of tissue fibrosis. Modulation of members of the av integrin family has exhibited profound effects on fibrosis in multiple organs and disease states. As reported, modulation of the subunit of av, which forms heterodimers with the $\beta 1, \beta 3, \beta 5, \beta 6$, and $\beta 8$ subunits, has exhibited profound effects on fibrosis in multiple organs and disease states [7-11]. Moreover, specifically depleting integrin av in HSCs could inhibit the progression of hepatic fibrosis, which strongly verified the vital function of integrin av in fibrosis [12]. At the same time, integrin av has been demonstrated to play a key role in the activation of latent TGF- $\beta 1$ through interacting with a linear arginine-glycine-aspartic acid (RGD) motif present in the LAP

\footnotetext{
${ }^{1} \mathrm{NHC}$ Key Laboratory of Biotechnology of Antibiotics, Institute of Medicinal Biotechnology, Chinese Academy of Medical Sciences, Beijing 100050, China; ${ }^{2}$ The Joint Program in

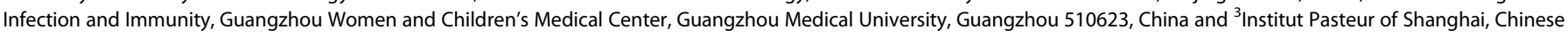
Academy of Sciences, Shanghai 200031, China

Correspondence: Ju-xian Wang (imbjxwang@163.com) or Hong-wei He (hehwei@imb.pumc.edu.cn)

These authors contributed equally: Na Zhang, Shuang-shuang Zhao
}

Received: 18 July 2019 Accepted: 31 October 2019

Published online: 13 January 2020 
662

[13]. Therefore, the therapeutic targeting of specific integrin av represents a promising avenue for treating a broad range of fibrotic diseases.

In our previous studies, we identified a novel biphenyl compound, IMB-S7, through our collagen type I a 1 (COL1A1) promoter screening model [14]. IMB-S7 (S7) is derived from bifendate (biphenyldicarboxylate), which is used for the treatment of chronic hepatitis in China $[15,16]$. In this article, we aimed to explore the antifibrotic potential of IMB-S7 and its related mechanisms.

\section{MATERIALS AND METHODS}

Reagents and antibodies

IMB-S7 was synthesized autonomously in our laboratory, and its purity was more than 98\% (HPLC). The pcDNA3.1-KLF5 plasmid was purchased from Youbio (Changsha, China), and pCMV3-MycITGAV was purchased from Sino Biological Inc. (Beijing, China); pcDNA3.1-SP1 was kindly provided by Dr Li Wang (Institute of Medicinal Biotechnology, China). Real-time PCR master mix was purchased from Roche (Indianapolis, USA).

Integrin av antibody was purchased from BD Biosciences (Becton, USA). Antibodies against Sp1, phosphor-Smad2/3, Smad2, and GAPDH were purchased from Cell Signaling Technology (Danvers, USA). Anti-a-SMA and anti-COL1A1 antibodies were obtained from Abnova (Taipei, Chinese Taiwan). Anti-TGF- $\beta 1$ antibody and recombinant TGF- $\beta 1$ protein were acquired from R\&D Systems (Minneapolis, USA).

Cell culture and Western blot

Human hepatic stellate LX2 cells were cultured in DMEM/ GlutaMAX I medium (Invitrogen, USA) with 10\% FBS and 1\% penicillin/streptomycin and incubated in a humidified atmosphere with $5 \% \mathrm{CO}_{2}$ at $37^{\circ} \mathrm{C}$. No mycoplasma contamination was found in LX2 cells using DAPI staining.

For Western blot analysis, cells were washed twice with ice-cold PBS and lysed in RIPA buffer supplemented with protease inhibitor cocktail (Roche). Proteins were separated by SDS-PAGE and transferred to PVDF membranes. The membranes were probed with the appropriate primary antibodies and an HRP-conjugated secondary antibody. Finally, blots were visualized using the Tanon 5200 system (Tanon, Shanghai, China). GAPDH was used as the internal control.

Small interfering RNA (siRNA) knockdown and transfection LX2 cells were transfected with commercially available siRNA targeting integrin av and Sp1 (Santa Cruz) following the manufacturer's instructions. One day before transfection, the medium of subconfluent LX2 cells was replaced with fresh DMEM/GlutaMAX I medium without penicillin/streptomycin. The cells were incubated with a complex formed by siRNA $(50 \mathrm{nM})$, transfection reagent (Lipofectamine RNAiMAX, Invitrogen), and transfection medium (Opti-MEM I, Invitrogen) for $48 \mathrm{~h}$ at $37^{\circ} \mathrm{C}$. A scrambled siRNA sequence (Santa Cruz proprietary target sequence) was used as a control. The transfection efficiency was confirmed by Western blot, as described above.

Real-time PCR

Total RNA from LX2 cells or liver tissue samples was extracted using TRIzol reagent. Complementary DNA (cDNA) was generated using a Transcriptor First Strand cDNA Synthesis Kit (Roche). The relative expression levels of specific genes were determined with an $A B I 7500$ Fast Real-Time PCR system (Thermo Fisher, USA). Data were calculated by the cycle threshold $\left({ }^{\triangle \triangle} \mathrm{CT}\right)$ method and normalized to GAPDH. The primers for the target genes were obtained from Applied Biosystems (Foster City, USA).
Animal experiments

Male adult Sprague-Dawley rats weighing 200-230 g were purchased from the Laboratory Animal Center (Academy of Military Medical Sciences, China). The rats were divided randomly into three groups: the sham group $(n=6)$, bile duct ligation (BDL) group $(n=6)$, and IMB-S7 group $(n=6)$. In the sham group, a 1-2$\mathrm{cm}$ midline incision in the abdomen was made and then closed. In the BDL and IMB-S7 groups, the bile duct was doubly ligated using 5-0 silk sutures after midline laparotomy and transected at $0.7-0.8 \mathrm{~cm}$ distal to the last bifurcation. One day after surgery, the rats in the IMB-S7 group were given $400 \mathrm{mg} / \mathrm{kg} \mathrm{IMB-S7} \mathrm{by} \mathrm{oral}$ administration for 14 days. The rats in the BDL group were given saline as a control. On the last day, the rats were sacrificed under anesthesia by $\mathrm{CO}_{2}$ after an overnight fast. Serum, urine, liver samples, and bile of the BDL and IMB-S7 groups were collected for further analyses. All animal experiments were approved by the Institutional Animal Care and Use Committee of the Institute of Medicinal Biotechnology \& Chinese Academy of Medical Sciences.

Serum, urine, and bile biochemistry

Serum alanine aminotransferase (ALT), aspartate aminotransferase (AST), total bilirubin (TBIL), cholesterol (CHO), low-density lipoprotein cholesterol (LDL), high-density lipoprotein cholesterol (HDL), and total bile acid (TBA); urine TBIL and TBA; and bile TBIL and TBA values were analyzed using a Hitachi 7170 chemistry analyzer with detection kits from Zhongsheng Beikong Biotechnology (Beijing, China) according to the manufacturer's instructions.

\section{Liver histology and tissue biochemistry}

Formalin-fixed liver tissue was embedded in paraffin and stained with hematoxylin and eosin (H\&E) or Sirius red. Liver bile duct proliferation and necrosis were quantified on a 1 to 5 scale in a blinded manner using a Leica DM1000 microscope. Histological sections from each animal were observed at low magnification ( $\times 10$ objective lens, Olympus, IX73) in a blinded manner to analyze the percentage of the fibrotic area. The contents of hydroxyproline liver tissues were detected utilizing kits from Nanjing Jiancheng Bioengineering Institute (Nanjing, China) according to the manufacturer's instructions.

Mouse primary HSC precipitation

Mouse primary HSCs were isolated according to the protocol of Ingmar Mederacke [17]. The process includes three sequential stages: (i) in situ perfusion of mouse liver through the hepatic portal vein with EGTA, pronase, and collagenase IV solutions; (ii) subsequent in vitro digestion with pronase/collagenase IV/DNase I for $20 \mathrm{~min}$; and (iii) density gradient-based separation of HSCs from other hepatic cell populations in the presence of $18 \%$ Nycodenz stock solution. Cell purity was observed by fluorescence microscopy and determined by immunofluorescence staining for desmin. Primary HSCs were cultured in DMEM with $10 \%$ FBS and $1 \%$ penicillin/streptomycin.

Dual luciferase reporter assay

The reporter construct contained the ITGAV promoter spanning the region of $-2297 \mathrm{bp}$ to $+152 \mathrm{bp}$. The region was amplified using PCR from the genomic DNA of LX2 cells and cloned into the pGL4.20 basic vector at the Kpnl and Bglll sites (Promega, USA), and the constructed reporter was named pGL4.20-2297. In addition to pGL4.20-2297, four short luciferase reporter variants, including pGL4.20-1295 (-1295 to +152), pGL4.20-796 (-796 to +152$)$, pGL4.20-309 (-309 to +152), and pGL4.20-16 $(-16$ to +152$)$, were constructed as above. In the construction, the following primers were used:

integrin av/Kpnl -2297: 5'-GGCCGGTACCTCCAGGTAGACTGGT TGTCATGT-3'; integrin av/Kpnl -1295: 5'-GGCCGGTACCGTCCACA CAATGCACTTAAA-3'; 
integrin av/Kpnl -796: 5'-TTGGTACCGCAAGAGGCTATGCT-3'; integrin av/Kpnl -309: 5'-TाTGGTACCCCTCCTTCCAGGTCTCCT CC-3';

integrin av/Kpnl -16: 5'-AACCAGATCTAGCTCCTGAGCCTG-3'; integrin av/Bg/ll + 152: 5'-GGCCAGATCTACTGTCCACGTCTAGG TTGAAGG-3'. Each construct together with pRL-TK was transfected into LX2 cells. The promoter activity was detected using the dual luciferase assay system (Promega) according to the manufacturer's instructions.

DNA affinity precipitation assay (DAPA)

The $5^{\prime}$ biotin end-labeled sense and antisense oligonucleotides of TGCGCTGCTGTCCCCGCCCCGCGCGCTCTG, which contain the binding sequence TCCCCGCCCCG $(-173 /-163 \mathrm{bp})$ of the ITGAV promoter, were custom made by Life Technologies. The $5^{\prime}$ biotin end-labeled sense and antisense oligonucleotides TGCGCTGC TGCGCGCTCTG, which do not have the binding sequence, were used as a negative control. Nuclear extract from LX2 cells was preincubated with DAPA buffer $(60 \mathrm{mM} \mathrm{KCl} ; 25 \mathrm{mM} \mathrm{HEPES}, \mathrm{pH} 7.6$; $5 \mathrm{mM} \mathrm{MgCl} 2 ; 7.5 \%$ glycerol; $0.1 \mathrm{mM}$ EDTA; $1 \mathrm{mM}$ DTT; and $0.25 \%$
Triton X-100) for $30 \mathrm{~min}$ on ice. One micromolar of biotin-labeled ds oligonucleotides was added for preincubation and further incubated for $45 \mathrm{~min}$ on ice. Then, the DNA/protein complexes were incubated with neutravidin-coated agarose beads (Pierce Chemical Co., IL) that were preequilibrated in DAPA buffer for $1 \mathrm{~h}$ at $4{ }^{\circ} \mathrm{C}$. Complexed proteins were examined by Western blot analysis with anti-Sp1 antibody [18].

\section{Statistical analysis}

All experiments were repeated at least three times, and the data are presented as the means $\pm S D$. Differences between groups were assessed for significance with two-tailed Student's $t$ tests. $P<0.05$ indicated significant differences between groups.

\section{RESULTS}

IMB-S7 ameliorates BDL-induced liver injury in rats

After IMB-S7 (Fig. 1a) administration for 14 days, several biochemical hepatic fibrosis indices were analyzed and are shown in Table 1. The serum levels of ALT and AST in the BDL group were a<smiles>COc1cc(O)c2c(c1)OCO2</smiles>

b

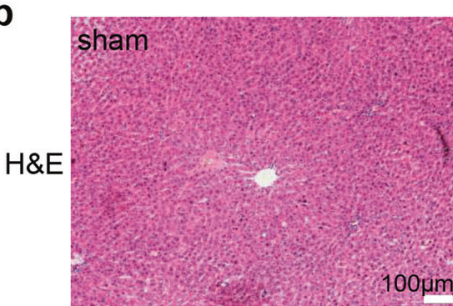

e

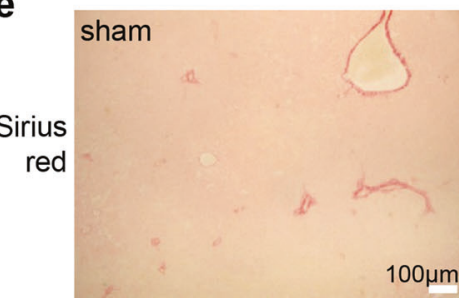

C

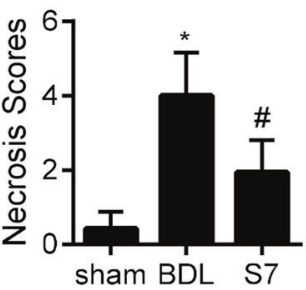

d

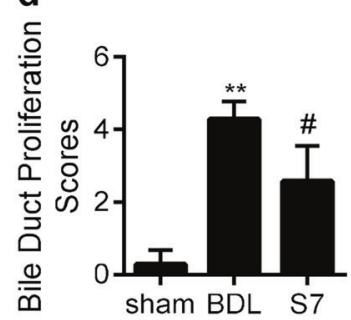


Table 1. Serum, urine, and bile biochemistry of bile duct ligation rats.

\begin{tabular}{|c|c|c|c|}
\hline & Sham $(n=6)$ & $\mathrm{BDL}(n=6)$ & IMB-S7 $(n=6)(400 \mathrm{mg} / \mathrm{kg})$ \\
\hline Serum AST (U/L) & $96.60 \pm 21.38$ & $700.33 \pm 133.51^{* *}$ & $485.67 \pm 96.15^{\# \#}$ \\
\hline Serum CHO (mmol/L) & $1.73 \pm 0.39$ & $2.5 \pm 0.81$ & $2.56 \pm 0.66$ \\
\hline Serum LDL (mmol/L) & $0.26 \pm 0.10$ & $1.15 \pm 0.35^{* *}$ & $1.34 \pm 0.38$ \\
\hline Serum HDL (mmol/L) & $1.18 \pm 0.25$ & $0.32 \pm 0.11^{* *}$ & $0.35 \pm 0.14$ \\
\hline Urine TBIL $(\mu \mathrm{mol} / \mathrm{L})$ & $3.12 \pm 4.29$ & $1159.74 \pm 301.78^{* *}$ & $1091.31 \pm 744.81$ \\
\hline Urine TBA $(\mu \mathrm{mol} / \mathrm{L})$ & $39.87 \pm 44.23$ & $4322.41 \pm 1705.77^{* *}$ & $4728.94 \pm 3262.25$ \\
\hline Bile TBIL $(\mu \mathrm{mol} / \mathrm{L})$ & No applicable & $268.74 \pm 262.03$ & $255.65 \pm 213.38$ \\
\hline
\end{tabular}

significantly elevated compared with the sham group, indicating the generation of a successful rat model. A statistical reduction in ALT and AST was observed after treatment with IMB-S7 (400 mg/ $\mathrm{kg}$ ). No significant differences in LDL, TBIL, and TBA were found between the BDL and IMB-S7 groups, suggesting that IMB-S7 might not influence bile acid synthesis or transportation. H\&E staining demonstrated that the histological structures of the BDL group were severely damaged, accompanied by extensive parenchyma necrosis and newly formed bile ducts (Fig. 1b). After IMB-S7 treatment, the pathological changes were substantially reduced (Fig. 1b). Blinded assessment showed that the IMB-S7 group had significantly lower scores for parenchymal necrosis and bile duct proliferation than the BDL group (Fig. 1c, d). Next, the antifibrotic effect of IMB-S7 was further assessed by Sirius red staining. In BDL rats, Sirius red-positive collagen fibrils extended not only to the portal areas but also to the hepatic parenchyma. However, collagen accumulation was strongly attenuated after IMB-S7 treatment (Fig. 1e, f). In addition, we observed that IMB-S7 dramatically reduced the hydroxyproline expression in the liver tissues caused by the BDL operation (Fig. 1g). Taken together, these data indicated that IMB-S7 can alleviate BDL-induced liver injury in rats.

IMB-S7 significantly inhibits HSC activation in cell models and rat fibrotic livers

To further verify the liver protective activity of IMB-S7, we first detected its effect on the TGF- $\beta /$ Smad pathway, whose activation promotes the transcription of most fibrogenesis-associated genes. SRB analysis revealed that IMB-S7 had little cytotoxicity in LX2 cells even at a relatively high concentration of $0.5 \mathrm{mM}$ (Fig. 2a). Therefore, the nontoxic concentrations of 0.2 and $0.5 \mathrm{mM}$ were chosen in the following studies. TGF- $\beta 1(2 \mathrm{ng} / \mathrm{mL})$ was used to preactivate LX2 cells for $24 \mathrm{~h}$, followed by IMB-S7 ( 0.2 and $0.5 \mathrm{mM})$ treatment for another $24 \mathrm{~h}$. The relative mRNA levels and protein expression of several biomarkers of activated HSCs, including TGF$\beta 1, \operatorname{COL} 1 \mathrm{~A} 1$, and $\mathrm{a}-\mathrm{SMA}$ (encoded by $A C T A 2$ ), were largely repressed by IMB-S7 (Fig. 2b, C). In addition, the phosphorylation of downstream Smad2/3 was also reduced by IMB-S7 (Fig. 2c). Consistent results were observed in rat liver tissues (Fig. 2d, e), primary mouse HSCs (Fig. 2f, g), and the rat hepatic stellate cell line HSC-T6 (data not shown). Altogether, these findings indicated that IMB-S7 could inhibit HSC activation in both cell models and animal models, which confirmed its inhibition of hepatic fibrosis progression.

IMB-S7 inhibits HSC activation through integrin av

As a subunit of the integrin family, integrin av is essential for HSC activation. We first examined integrin av activity after IMB-S7 treatment. In normal or TGF- $\beta 1$-activated LX2 cells, the mRNA and protein levels of integrin av were both reduced after IMB-S7 treatment (Fig. 3a, b). Furthermore, IMB-S7 administration also reversed the integrin av upregulation in BDL rats (Fig. $3 c$, d). These data suggested that integrin av expression was negatively regulated by IMB-S7. In order to confirm the role of integrin av in IMB-S7 regulation, we used siRNA-ITGAV to knockdown its expression in LX2 cells. Similar to IMB-S7 administration, the activation of TGF- $\beta 1, C O L 1 A 1, a-S M A$, and $p-S m a d 2 / 3$ was suppressed by siRNA-ITGAV (Fig. 3e). In addition, we further overexpressed integrin av in LX2 cells using pCMV3-Myc-ITGAV. As expected, the inhibitory effect of IMB-S7 was reversed by integrin av overexpression (Fig. 3f). As evidenced by knockdown and overexpression, integrin av may be a target of IMB-S7 in HSC activation, and the effect of IMB-S7 on repressing integrin av may contribute to the suppression of HSCs and consequently hepatic fibrosis.

IMB-S7 regulates the expression of integrin av at the $-309 /-16$ bp promoter region

To study the underlying mechanism of integrin av expression in response to IMB-S7 treatment, we first examined changes in the transcriptional activity of ITGAV. We constructed the ITGAV promoter (full length of $2297 \mathrm{bp}$ ) with the luciferase reporter gene using the pGL4.20 vector and named it pGL4.20-2297. ITGAV promoter activity was reflected by the fluorescence intensity. We transfected LX2 cells with pGL4.20-2297 and the control vector pGL4.20, and the fluorescence results showed that ITGAV promoter activity was suppressed by IMB-S7 (Fig. 4a). To find the accurate promoter region on which IMB-S7 acts, we transfected LX2 cells with pGL4.20-2297 and four truncation mutants (pGL4.20-1295, pGL4.20-796, pGL4.20-309, and pGL4.20-16). Based on the luciferase intensity, we observed that IMB-S7 induced a significant decrease in the pGL4.20-2297, pGL4.20-1295, pGL4.20-796, and pGL4.20-309 groups. However, when LX2 cells were transfected with pGL4.20-16 in which the region between -309 bp and -16 


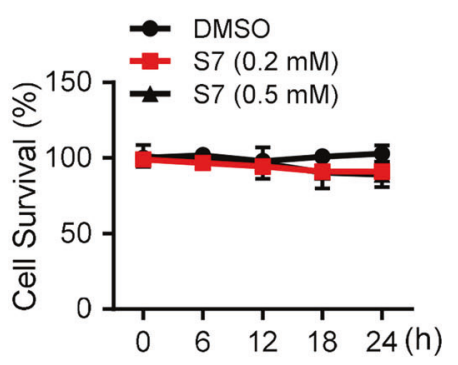

b

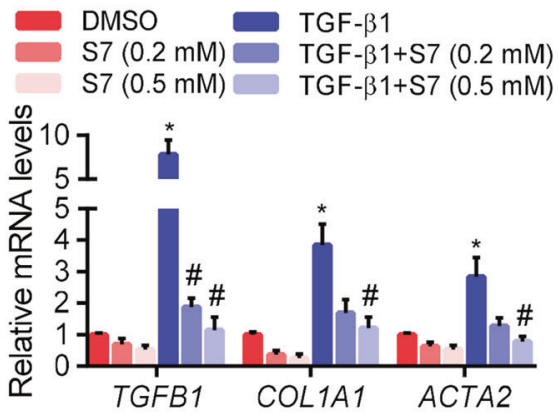

d

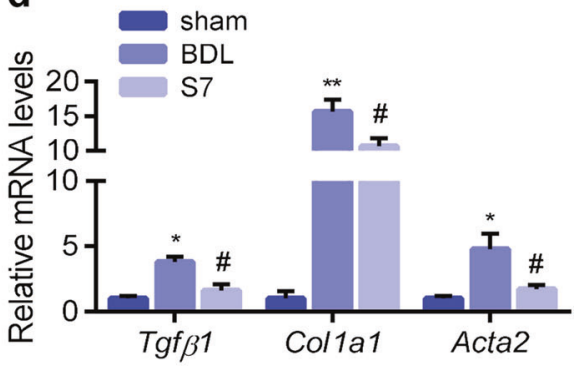

e

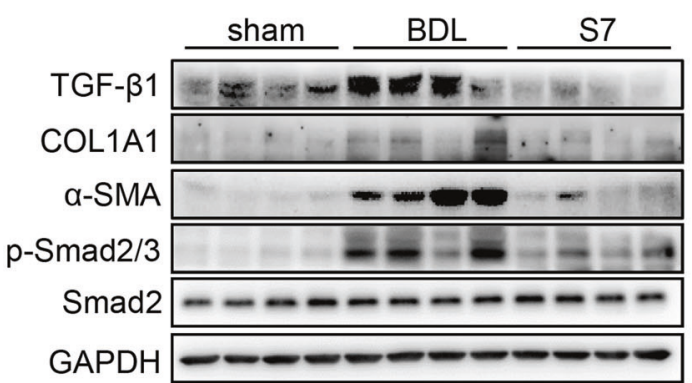

f

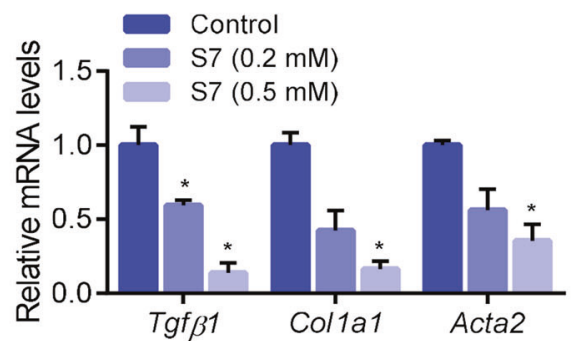

g $\mathrm{S} 7(\mathrm{mM}) \quad 0 \quad 0.20 .5$

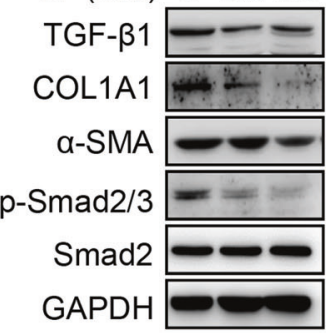

Fig. 2 IMB-S7 significantly inhibits hepatic fibrosis gene expression in HSCs and the BDL rat model. a LX2 cells were treated with different concentrations of IMB-S7 for the indicated times, and cell viability was measured by the sulforhodamine B (SRB) assay. b, c LX2 cells were starved for $24 \mathrm{~h}$ and then treated with TGF- $\beta 1(2 \mathrm{ng} / \mathrm{mL})$ and IMB-S7 $(0.2 \mathrm{mM}$ or $0.5 \mathrm{mM})$ for an additional $24 \mathrm{~h}$. Real-time PCR for TGFB1, COL1A1, and ACTA2 (b) and Western blot analysis for TGF- $\beta 1$, COL1A1, $\alpha-S M A, p-S m a d 2 / 3$, and Smad2 (c), ${ }^{*} P<0.05$ vs the DMSO group; ${ }^{\#} P<$ 0.05 vs the TGF- $\beta 1$ group. $\mathbf{d}$, e The mRNA and protein as in $\mathbf{b}$ and $\mathbf{c}$ in liver samples of the BDL rat model were also detected by real-time PCR and Western blot ( $n=6$ per group), ${ }^{*} P<0.05,{ }^{* *} P<0.01$ vs the sham group; ${ }^{*} P<0.05$ vs the BDL group. Mouse primary HSCs were treated with IMB-S7 (0.2 or $0.5 \mathrm{mM}$ ) for $24 \mathrm{~h}$. Real-time PCR for Tgf $\beta 1$, Col1 a 1, and Acta2 (f) and western blot analysis for TGF- $\beta 1$, COL1A1, $\alpha-S M A, p-S m a d 2 / 3$ and Smad2 (g). ${ }^{*} P<0.05$ vs the control group.

bp was missing, IMB-S7-mediated downregulation of the ITGAV promoter was completely abolished (Fig. 4b, pGL4.20-309 vs pGL4.20-16). These results suggested that IMB-S7 inhibits integrin av activity at the transcriptional level and that the regulatory interaction typically occurs in the region between $-309 \mathrm{bp}$ and $-16 \mathrm{bp}$ of the ITGAV promoter.
Sp1 plays a critical role in IMB-S7-mediated integrin av regulation To determine how the $-309 /-16$ bp promoter region is regulated by IMB-S7, we used the JASPAR database (http:// jaspar.genereg.net/), which specializes in predicting transcription factor binding profiles. Among the predicted factors, the KLF and $\mathrm{Sp}$ families score highest, especially the subunits of KLF5 and Sp1 
a

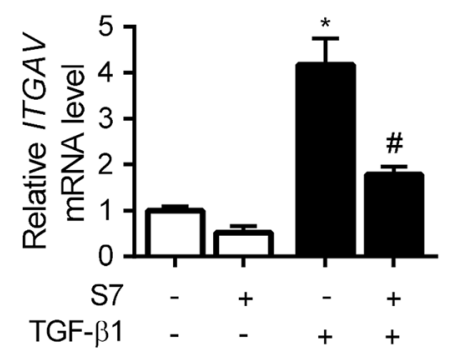

C

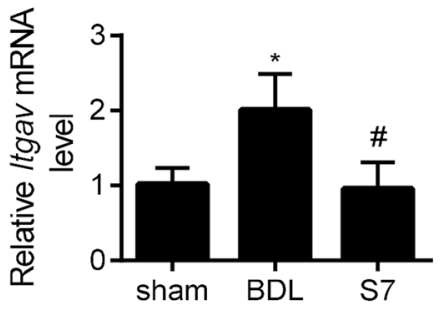

e

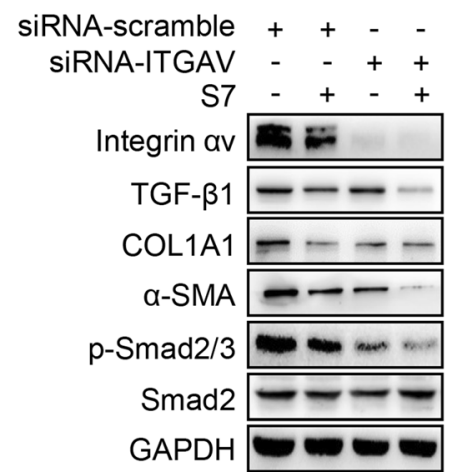

b

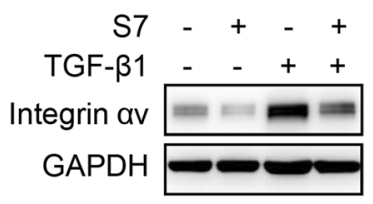

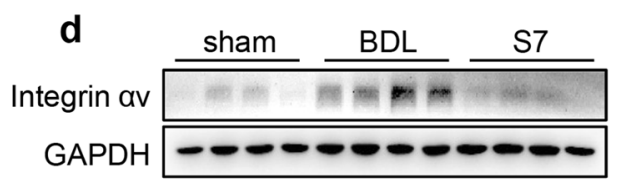

f

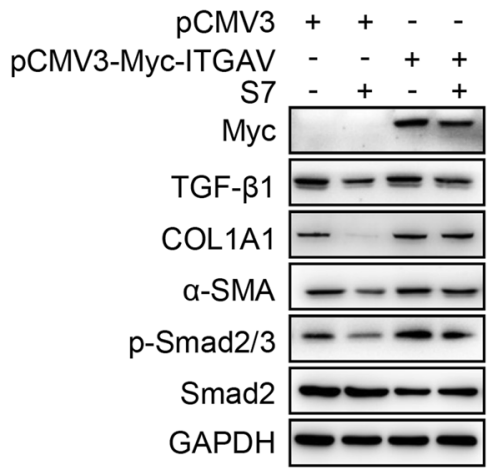

Fig. 3 IMB-S7 suppresses the expression of integrin av. a, b LX2 cells were starved for $24 \mathrm{~h}$ and then treated with TGF- $\beta 1$ ( $2 \mathrm{ng} / \mathrm{mL})$ and IMBS7 $(0.2 \mathrm{mM})$ for an additional $24 \mathrm{~h}$. Real-time PCR (a) and Western blot analysis (b) for integrin $\alpha \mathrm{v}^{*}{ }^{*} P<0.05$ vs the left column; ${ }^{\#} P<0.05$ vs the TGF- $\beta 1$ column. Real-time PCR (c) and Western blot analysis (d) for integrin $\alpha v$ in liver samples of the BDL rat model ( $n=6$ per group), ${ }^{*} P<0.05$ vs the sham group; ${ }^{*} P<0.05$ vs the BDL group. LX2 cells were plated and cultured in six-well plates overnight, followed by siRNA-ITGAV (e) or pCMV-Myc-ITGAV (f) for $48 \mathrm{~h}$, followed by IMB-S7 $(0.2 \mathrm{mM})$ for an additional $24 \mathrm{~h}$. Western blot analysis for the indicated proteins.

(data not shown). Furthermore, at the $-309 /-16$ bp region of the ITGAV promoter, we found the typical binding sites of KLF5 and Sp1 (Fig. 5a, the red bases indicate KLF5 binding sites, and the green bases indicate Sp1 binding sites). To further distinguish which transcription factor is the critical factor, we overexpressed pcDNA3.1-SP1 and pcDNA3.1-KLF5 in LX2 cells. Elevated mRNA levels of ITGAV were observed in the group that overexpressed SP1 but not KLF5 (Fig. 5b). Thus, we speculated that the transcription factor Sp1 participated in IMB-S7-mediated regulation. To confirm the function of Sp1, we performed overexpression and knockdown experiments. The downregulation of IMB-S7 on integrin av expression was partially reversed by Sp1 overexpression (Fig. 5c). At the same time, we used a specific siRNA targeting Sp1 to knockdown its expression. We observed that the downregulation of IMB-S7 on integrin av was further enhanced (Fig. 5d).

Furthermore, to examine how Sp1 mediates integrin av expression, we performed a DAPA experiment to assess whether IMB-S7 could influence the direct binding of Sp1 to the ITGAV promoter. To simulate the binding as accurately as possible, we used biotin-labeled 5'-TGCGCTGCTGTCCCCGCCCCGCGCGCTCTG-3' $(-183 /-153 \mathrm{bp})$ oligonucleotides (biotin-Sp1), including the binding site 5'-TCCCCGCCCCG-3' $(-173 /-163$ bp) of Sp1. The biotin-labeled 5'- TGCGCTGCTGCGCGCTCTG-3' oligonucleotides (biotin-control), excluding the Sp1 binding site, were used as a negative control. As expected, Sp1 was pulled down together with the oligonucleotides in the biotin-Sp1 group, indicating that Sp1 could interact directly with the ITGAV promoter (Fig. 5e). Furthermore, after the addition of IMB-S7, the binding effect was significantly repressed (Fig. 5e). Our results indicated that IMB-S7 functions as a fibrotic inhibitor by repressing Sp1 binding to the ITGAV promoter.

Together, we first assessed the novel biphenyl compound IMBS7 in this article, and our study comprehensively confirmed its in vivo and in vitro antifibrotic activity. We reported that IMB-S7 inhibits HSC activation and liver fibrosis through Sp1-integrin av signaling.

\section{DISCUSSION}

Hepatic fibrosis, as an unavoidable process from liver injury to cirrhosis and even liver cancer, is a dynamic process that has an inherent capacity for recovery and remodeling [19], and much has been learned about the pathophysiology of hepatic fibrosis in recent decades. However, new and effective agents for the treatment of hepatic fibrosis are urgently needed. 

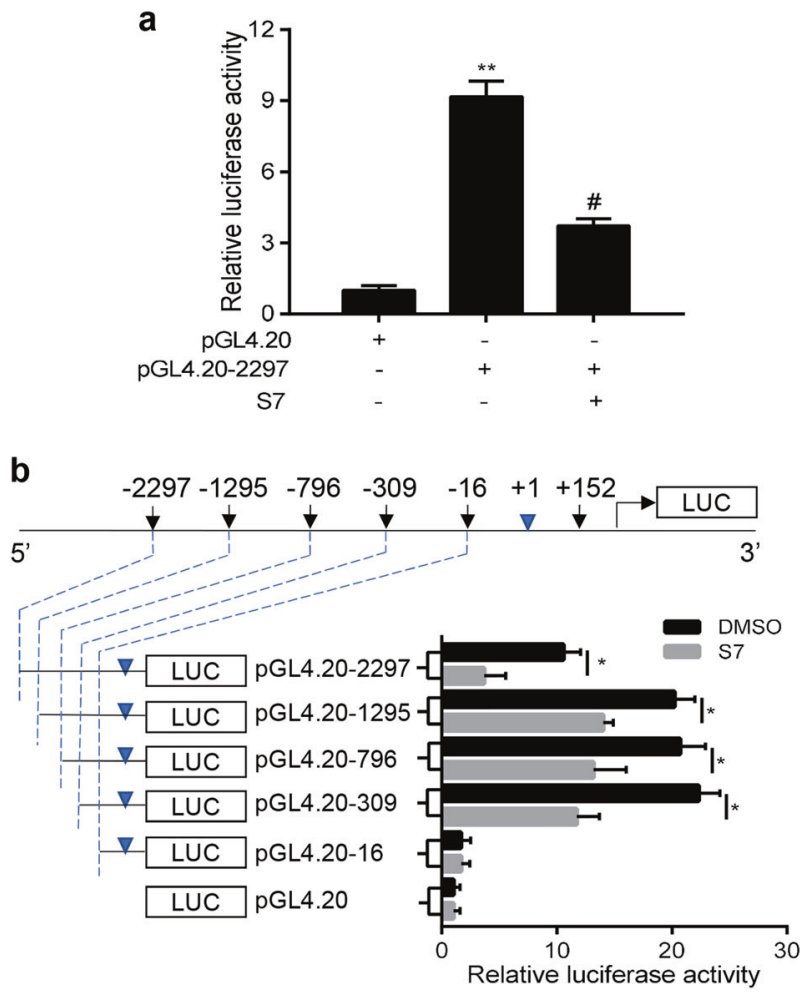

Fig. 4 IMB-S7 suppresses the promoter activity of ITGAV. a LX2 cells were transfected with pGL4.20-2297 plasmid for $24 \mathrm{~h}$, followed by incubation with IMB-S7 $(0.2 \mathrm{mM})$ for another $24 \mathrm{~h}$, and the ITGAV promoter activity was determined by the dual luciferase reporter assay. The data are expressed as the mean $\pm S D$, ${ }^{* *} P<0.01$ compared with the pGL4.20 group; ${ }^{\#} P<0.05$ compared with the pGL4.20-2297 group. $\mathbf{b}$ LX2 cells were transfected with pGL4.20-2297 and different truncations (pGL4.20-1295, pGL4.20-796, pGL4.20-309, and pGL4.20-16) for $24 \mathrm{~h}$, followed by IMB-S7 $(0.2 \mathrm{mM})$ incubation for $24 \mathrm{~h}$, and the activity of different promoters was determined by the dual luciferase reporter assay. Data are shown as a fold-induction relative to the pGL4.20 group. The data are expressed as the mean \pm $\mathrm{SD},{ }^{*} P<0.05$ compared with the corresponding DMSO group.

Bifendate (DDB), an intermediate in the synthesis of schizandrin, is an anti-HBV drug used in Chinese medicine for the treatment of chronic hepatitis $B$ and is widely used to treat patients with elevated transaminase caused by viral hepatitis and drug-induced liver injury $[15,20]$. However, it cannot improve the pathological changes in chronic hepatitis, and its antifibrotic effect has seldom been reported. Thus, we modified the structure of DDB to identify candidates for antifibrosis. From dozens of candidates, we identified IMB-S7 as a potential chemical compound for the treatment of liver fibrosis by the highthroughput drug screening model based on COL1A1 promoter activity established in our laboratory [14]. The following verification in vivo and in vitro confirmed its remarkable antifibrotic effect.

In BDL rats, the elevated serum activities of AST and ALT caused by $\mathrm{BDL}$ were significantly decreased after IMB-S7 supplementation. IMB-S7 significantly reduced necrosis and bile duct proliferation and markedly inhibited fibrogenesis as evidenced by $H \& E$ or Sirius red staining and the hydroxyproline content assay (Fig. 1). These observations suggest that IMB-S7 has a protective role against BDL-induced liver injury. In addition, the elevated inflammation-associated cytokines such as NF-KB and TNF- $\alpha$ in BDL rats were reduced by IMB-S7, and reduced superoxide dismutase in BDL rat livers was elevated by IMB-S7 (data not shown), which suggests a protective effect of IMB-S7 on liver inflammation and oxidative stress [20, 21]. However, IMB-S7 seems to have no impact on bile acids or bilirubin, although a bile duct ligation model was employed. We then speculated that IMBS7 regulates liver fibrosis in other ways instead of impacting bile acids or bilirubin.

TGF- $\beta 1$ has been characterized as one of the key cytokines that mediate HSC activation and hepatic fibrogenesis [22]. The physiological and pathological activities of TGF- $\beta 1$ were propagated by the canonical TGF- $\beta / S$ mad pathway to modulate gene expression [23]. Thus, we then detected the effect of IMB-S7 on TGF- $\beta$ and the classic Smad signaling pathway. In our study, IMB-S7 dramatically reduced TGF- $\beta 1$ expression and the TGF- $\beta /$ Smad signaling pathway activity both in vivo and in vitro (Fig. 2). Activation of TGF- $\beta 1$ requires the binding of integrin av to an RGD sequence in the prodomain [24]. Integrin av was identified as a core molecular pathway that regulates fibrosis in several organs [12]. These reports and our observations attracted our attention, and we examined whether IMB-S7 affects integrin av, which may result in the antihepatic fibrosis effect of IMB-S7. As expected, the mRNA and protein levels of integrin av were both reduced significantly by IMB-S7 both in vitro and in vivo (Fig. 3). Interestingly, we also found that ITGAV knockdown dramatically suppressed TGF- $\beta /$ Smad signaling pathway activation, which was similar to the effects of IMB-S7, and the inhibitory effect of IMB-S7 was neutralized by ITGAV overexpression. Thus, we hypothesized that IMB-S7 suppressed the TGF- $\beta$ / Smad signaling pathway through integrin av.

How does IMB-S7 regulate integrin av mRNA? Does IMB-S7 act on ITGAV mRNA directly? To answer this question, we constructed a recombinant plasmid with the ITGAV promoter and a luciferase reporter gene. The luciferase reporter system showed that IMB-S7 reduced ITGAV promoter activity in vitro, indicating that IMB-S7 directly acts on ITGAV (Fig. 4). The ITGAV promoter truncation assay finally focused our attention on the $-309 /-16 \mathrm{bp}$ fragment, and the JASPAR database predicted that the $\mathrm{Sp}$ and KLF families are the main transcription factors that bind to this region. Overexpression or knockdown of Sp1 or KLF5 confirmed the role of Sp1, but not KLF5, in the regulation of integrin av (Fig. 5). Sp1 has been implicated in the regulation of fibrosis in several previous studies. Wu et al. proved that silencing of $\mathrm{Sp} 1$ diminished the stimulation of integrin av expression by sulfatide [25], which agrees with our results. García-Ruiz et al. stated that mutation of Sp1 reversed leptininduced Col1a1 gene expression in primary rat HSCs [26]. DAPA analysis revealed that IMB-S7 reduced the direct interaction of Sp1 and the ITGAV promoter. However, we found that IMB-S7 not only regulated the binding of $\mathrm{Sp} 1$ to the ITGAV promoter but also decreased the Sp1 protein level (Fig. 5c, d), and the detailed regulatory mechanism of IMB-S7 on Sp1 needs further study.

In conclusion, our study demonstrated that IMB-S7 reduced the binding of $\mathrm{Sp} 1$ to the ITGAV $-173 \mathrm{bp}$ to -163 bp promoter region, contributing to the decreased integrin av expression in LX2 cells. Decreased expression of integrin av in turn leads to repression of the TGF- $\beta /$ Smad pathway. Therefore, our study not only identified a novel biphenyl compound, IMB-S7, as a great potential compound for the treatment of hepatic diseases but also elucidated the regulatory mechanism between $\mathrm{Sp} 1$ and integrin av in fibrosis. 
a

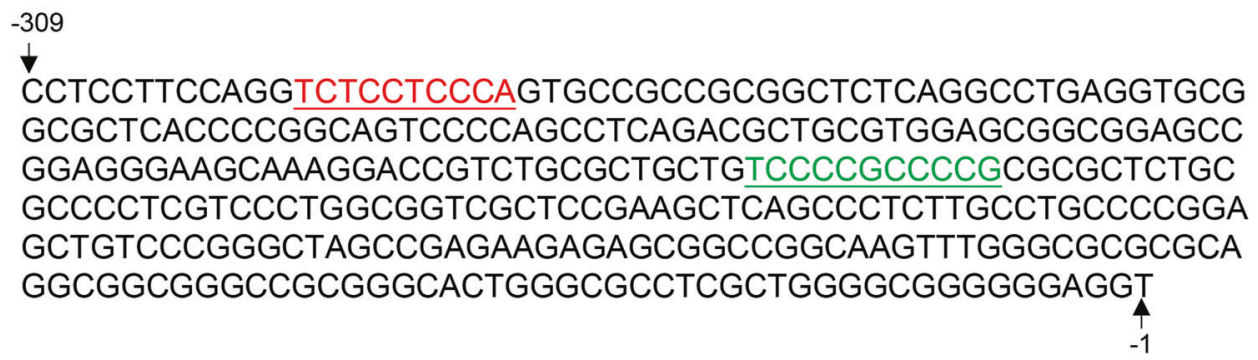

b
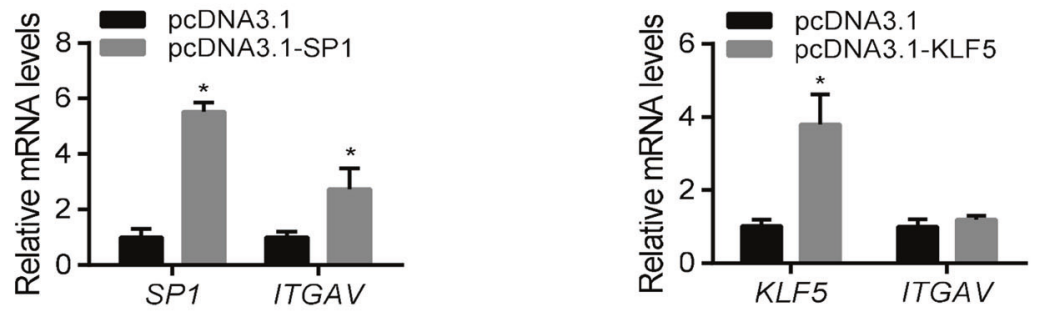

C

d
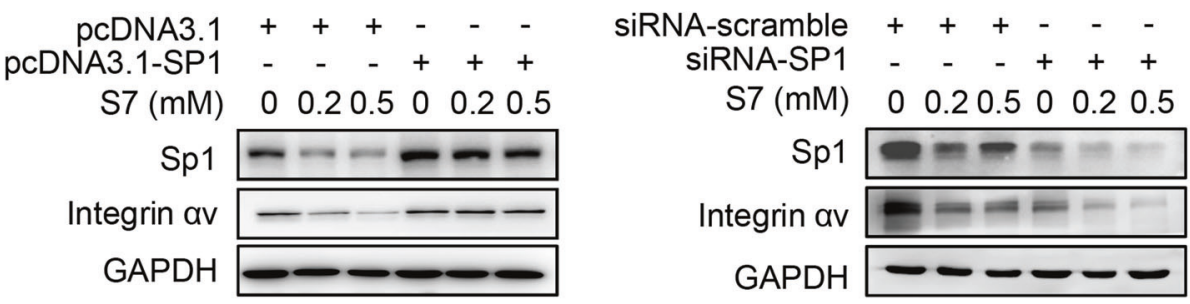

e

\begin{tabular}{rllll} 
S7 & - & + & - & + \\
Biotin-control & + & + & - & - \\
Biotin-Sp1 & - & - & + & + \\
\cline { 3 - 4 } Sp1 & & & - \\
\cline { 3 - 5 }
\end{tabular}

Biotin-control: TGCGCTGCTGCGCGCTCTG

Biotin-Sp1: TGCGCTGCTGTCCCCGCCCCGCGCGCTCTG

-173 GC-rich $\stackrel{4}{-163}$

Fig. 5 The transcription factor Sp1 regulates the promoter activity of ITGAV. a The representative binding site of KLF5 (red, TCTCCTCCCA) and Sp1 (green, TCCCCGCCCCG) on the $-309 /-16$ bp region of the ITGAV promoter. b LX2 cells were transiently overexpressed with pcDNA3.1-SP1 (left) or pcDNA3.1-KLF5 (right) for $48 \mathrm{~h}$, and real-time PCR for ITGAV and SP1 or KLF5. The data are expressed as the mean \pm SD, ${ }^{*} P<0.05$ compared with the pcDNA3.1 group. LX2 cells were transiently transfected with pcDNA3.1-SP1 (c) or siRNA-SP1 (d) for 48 h, followed by IMB-S7 for an additional $24 \mathrm{~h}$. Western blot analysis for Sp1 and integrin $\alpha \mathrm{v}$. e Biotin-labeled 30-mer GC-rich oligonucleotides (biotin-Sp1) were incubated with LX2 cell lysis in the presence of IMB-S7 or not, and Western blot analysis was performed for Sp1 that specifically bound to the biotin probes.

\section{ACKNOWLEDGEMENTS}

This work was supported by the Fundamental Research Funds for the Central Universities (3332019083), the National Natural Science Foundation of China (Nos. 81673497, 81903695), the CAMS Innovation Fund for Medical Sciences (No. 2019-12M1-001), and the National Science \& Technology Major Project "Key New Drug Creation and Manufacturing Program",China(No.2019ZX09201001).

\section{AUTHOR CONTRIBUTIONS}

HWH and RGS designed the experiments. SSZ performed animal experiments. YXZ and NZ performed the experiments on the cell model. JXW and YCW acquired and analyzed all data. NZ and SSZ wrote and revised the paper. Every author read and approved the paper.

\section{ADDITIONAL INFORMATION}

Competing interests: The authors declare no competing interests.

\section{REFERENCES}

1. Bataller R, Brenner DA. Liver fibrosis. J Clin Investig. 2005;115:209-18.

2. Schuppan D, Afdhal NH. Liver cirrhosis. Lancet. 2008;371:838-51. 
3. Hasan IH, El-Desouky MA, Hozayen WG, Abd el Aziz GM. Protective effect of zingiber officinale against $\mathrm{CCl}_{4}$-induced liver fibrosis is mediated through downregulating the TGF-beta1/Smad3 and NF-KB/IKB pathways. Pharmacology. 2016;97:1-9.

4. Zhang Y, Meng XM, Huang XR, Wang XJ, Yang L, Lan HY. Transforming growth factor-beta1 mediates psoriasis-like lesions via a Smad3-dependent mechanism in mice. Clin Exp Pharmacol Physiol. 2014;41:921-32.

5. Hynes RO. Integrins: bidirectional, allosteric signaling machines. Cell. 2002;110:673-87.

6. Pellicoro A, Ramachandran P, Iredale JP, Fallowfield JA. Liver fibrosis and repair: immune regulation of wound healing in a solid organ. Nat Rev Immunol 2014;14:181-94.

7. Munger JS, Huang X, Kawakatsu H, Griffiths MJ, Dalton SL, Wu J, et al. The integrin alpha $v$ beta 6 binds and activates latent TGF beta 1: a mechanism for regulating pulmonary inflammation and fibrosis. Cell. 1999;96:319-28.

8. Wang B, Dolinski BM, Kikuchi N, Leone DR, Peters MG, Weinreb PH, et al. Role of alphavbeta6 integrin in acute biliary fibrosis. Hepatology. 2007:46:1404-12.

9. Hahm K, Lukashev ME, Luo Y, Yang WJ, Dolinski BM, Weinreb PH, et al. Alphav beta6 integrin regulates renal fibrosis and inflammation in Alport mouse. Am J Pathol 2007;170:110-25.

10. Popov Y, Patsenker E, Stickel F, Zaks J, Bhaskar KR, Niedobitek G, et al. Integrin alphavbeta 6 is a marker of the progression of biliary and portal liver fibrosis and a novel target for antifibrotic therapies. J Hepatol 2008;48:453-64.

11. Conroy KP, Kitto $\amalg$, Henderson NC. Alphav integrins: key regulators of tissue fibrosis. Cell Tissue Res. 2016;365:511-9.

12. Henderson NC, Arnold TD, Katamura Y, Giacomini MM, Rodriguez JD, McCarty JH, et al. Targeting of alphav integrin identifies a core molecular pathway that regulates fibrosis in several organs. Nat Med 2013;19:1617-24.

13. Wipff PJ, Rifkin DB, Meister JJ, Hinz B. Myofibroblast contraction activates latent TGF-beta1 from the extracellular matrix. J Cell Biol 2007;179:1311-23.

14. Zhao SS, Wang JX, Wang YC, Shao RG, He HW. [Establishment and application of a high-throughput drug screening model based on COL1A1 promoter for antiliver fibrosis]. Yao Xue Xue Bao. 2015;50:169-73.
15. Cui S, Wang M, Fan G. Anti-HBV efficacy of bifendate in treatment of chronic hepatitis B, a primary study. Zhonghua Yi Xue Za Zhi. 2002;82:538-40.

16. Wu T, Roger H, Xie L, Liu G, Hao B. Bicyclol for chronic hepatitis B. Cochrane Database Syst Rev 2006;4:CD004480.

17. Mederacke I, Dapito DH, Affo S, Uchinami H, Schwabe RF. High-yield and highpurity isolation of hepatic stellate cells from normal and fibrotic mouse livers. Nat Protoc 2015;10:305-15.

18. Walker GE, Wilson EM, Powell D, Oh Y. Butyrate, a histone deacetylase inhibitor activates the human IGF binding protein-3 promoter in breast cancer cells: molecular mechanism involves an Sp1/Sp3 multiprotein complex. Endocrinology. 2001;142:3817-27.

19. Ellis EL, Mann DA. Clinical evidence for the regression of liver fibrosis. J Hepatol 2012;56:1171-80.

20. El-Beshbishy HA. The effect of dimethyl dimethoxy biphenyl dicarboxylate (DDB) against tamoxifen-induced liver injury in rats: DDB use is curative or protective. $J$ Biochem Mol Biol 2005;38:300-6.

21. Kim SG, Kim HJ, Choi SH, Ryu JY. Inhibition of lipopolysaccharide-induced IkappaB degradation and tumor necrosis factor-alpha expression by dimethyl4, $4^{\prime}$-dimethoxy-5,6,5',6'-dimethylene dioxybiphenyl-2,2'-dicarboxylate (DDB): minor role in hepatic detoxifying enzyme expression. Liver. 2000;20:319-29.

22. Akhurst RJ, Hata A. Targeting the TGFbeta signalling pathway in disease. Nat Rev Drug Discov 2012;11:790-811.

23. Kang Y. Pro-metastasis function of TGFbeta mediated by the Smad pathway. J Cell Biochem. 2006;98:1380-90.

24. Shi M, Zhu J, Wang R, Chen X, Mi L, Walz T, et al. Latent TGF-beta structure and activation. Nature. 2011:474:343-9.

25. Wu W, Dong YW, Shi PC, Yu M, Fu D, Zhang CY, et al. Regulation of integrin alphaV subunit expression by sulfatide in hepatocellular carcinoma cells. J Lipid Res 2013;54:936-52.

26. Garcia-Ruiz I, Gomez-Izquierdo E, Diaz-Sanjuan T, Grau M, Solis-Munoz P, MunozYague T, et al. Sp1 and Sp3 transcription factors mediate leptin-induced collagen alpha1(I) gene expression in primary culture of male rat hepatic stellate cells. Endocrinology. 2012;153:5845-56. 